\title{
Nanoscale Chemical Imaging in Zeolite Catalysts by Atom Probe Tomography
}

\author{
Jonathan Poplawsky ${ }^{1}$, Sophie Van Vreeswijk ${ }^{2}$, Joel Schmidt ${ }^{2}$, Matteo Monai ${ }^{2}$, Florian Zand ${ }^{2}$ and Bert Weckhuysen ${ }^{2}$ \\ ${ }^{1}$ Oak Ridge National Laboratory, Tennessee, United States, ${ }^{2}$ Utrecht University, United States
}

Zeolites are crystalline nanoporous materials that are most commonly used in oil refining and automotive emission treatments. Zeolites function by having internal acid sites, defined by an element's identity and lattice site (e.g. Brønsted or Lewis acid sites), that catalyze desired reactions. Different strategies are used to improve the performance of zeolites, such as inventing new structures or changing the active sites by exchanging elements. Engineering improved zeolite catalysts relies on understanding structure-composition-property relationships. This remains challenging because zeolite structure and composition heterogeneities span nanoscopic to microscopic length scales, while nanoscopic regimes are the most difficult to examine. Atom probe tomography (APT) has been proven to be an effective technique for probing the 3D composition of zeolites with sub-nm resolution.

Different zeolites can be used as catalysts for the methanol-to-hydrocarbons (MTH) reaction. During this reaction, aromatic hydrocarbon molecules can get stuck in the zeolites' molecular sieves, which degrades the performance. This is known as coking. APT has been used to understand nanoscale coking mechanisms within zeolite ZSM-5, SAPO-34, and SSZ-13.[1-3] ZSM-5 is a medium pore zeolite with an MFI structure, while SAPO-34 and SSZ-13 have the same small pore chabazite (CHA) structure. The zeolite frameworks are shown in Figure 1a. SAPO-34 and SSZ-13 differ in their framework elements, which changes the strength of the acid sites. APT found drastically different coke distributions within ZSM-5 compared to the CHA zeolites, while the CHA zeolites showed similar coke distributions despite their dissimilar acid sites. For example, obvious heterogeneities in the $\mathrm{C}$ content were found in ZSM-5 at $\mu \mathrm{m}$ and nm length scales for mildly coked to fully coked (deactivated) samples. For example, the APT dataset shown in Figure 1c shows a non-C containing region that spans several 10s of nms surrounded by a heavily coked region with $\mathrm{C}$ clusters that were extracted using the maximum separation method. These clusters were found to be linked to regions with higher $\mathrm{Al}$ contents, indicating that single molecules become stuck at individual atomic reaction sites. On other hand, the CHA zeolites only showed a slight deviation from random in the $\mathrm{C}$ nearest neighbor distribution for samples that were mildly coked. After longer reaction times resulting in a higher coke content, a deviation from random in the C-NND was undetectable. It is intuitive that $\mathrm{C}$ species are clustered at the nm length scale because they are bound to each other within coke molecules. The inability to identify C-NND heterogeneities in the CHA structure after significant coking is mainly due to the combination of the spatial uncertainty of APT, the small pore size resulting in small coke molecules, and the even, random distribution of coked pores. The larger ZSM-5 pores are less affected by the spatial uncertainties of APT, and clear clusters were able to be extracted. A demonstration of zeolite element spatial blurring in CHA is shown in Figure $1 \mathrm{~b}$.

Metal ion exchanges within zeolite frameworks are used to improve the catalytic performance or change the catalytic reaction. For example, Cu-exchanged ZSM-5 (Cu-ZSM-5) and Cu-SSZ-13 are used in the ammonia selective catalytic reduction $\left(\mathrm{NH}_{3}-\mathrm{SCR}\right)$ for $\mathrm{NO}_{\mathrm{x}}$ emissions. Cu-SSZ-13 demonstrates a markedly improved reactivity and stability compared to Cu-ZSM-5. APT was performed on fresh and aged (subject to a 135,000 mile simulation) Cu-ZSM-5 and Cu-SSZ-13.[4] The APT data for both fresh zeolites indicated $\mathrm{Cu}$ clustering and a homogenous $\mathrm{Al}$ distribution as indicated by radial distribution functions and iso-concentration surfaces. After the 135,000-mile industry standard simulation, both zeolites showed signs of $\mathrm{Cu}$ and $\mathrm{Al}$ motion and agglomeration; however, Cu-ZSM-5 showed a much higher degree of Al-Cu clustering than $\mathrm{Cu}-\mathrm{SSZ}-13$. Proximity histogram analysis revealed an $\mathrm{Al} / \mathrm{Cu}$ ratio of 2 in localized regions (a few nms) only for the aged $\mathrm{Cu}-\mathrm{ZSM}-5$ as shown in Figure 1d. This correlates to a spinel $\mathrm{Al}_{2} \mathrm{CuO}_{4}$ phase that has been long regarded as a deactivating species. Prior to this measurement, the $\mathrm{Al}_{2} \mathrm{CuO}_{4}$ phase has only been identified in aged $\mathrm{Cu}-\mathrm{ZSM}-5$ using bulk analysis techniques. The superior stability of Cu-SSZ-13 over $\mathrm{Cu}-\mathrm{ZSM}-5$ can be attributed to the more sluggish motion of $\mathrm{Cu}$ and $\mathrm{Al}$ during the $\mathrm{NH}_{3}-\mathrm{SCR}$ reaction. 
APT has facilitated the ability to probe zeolite materials at nano-length scales. C clusters with similar numbers of $\mathrm{C}$ atoms to known coke species have been identified after the MTH reaction for several zeolite materials, and the spatial affinity of coke molecules with individual Brønsted acid sites has been discovered. APT has also identified nanoscale non-active $\mathrm{Al}_{2} \mathrm{CuO}_{4}$ phases in $\mathrm{Cu}-\mathrm{ZSM}-5$ that absorb catalytically active $\mathrm{Cu}$ atoms, thereby, deactivating the catalyst. Overall, APT has demonstrated its value in understanding nanoscale catalytic reactions within conventional and cation exchanged zeolite frameworks. Both the challenges and successes in APT studies of zeolite materials will be discussed [5].
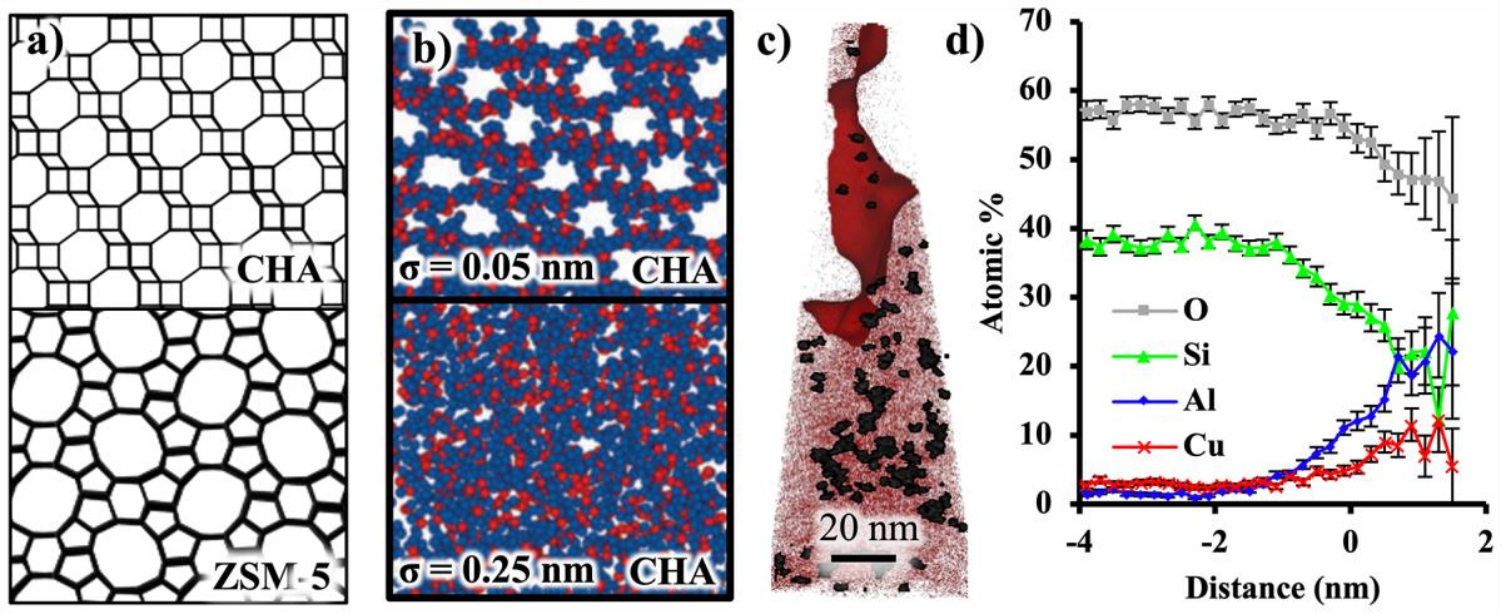

Figure 1. (a) The chabazite (CHA) and MFI (ZSM-5) zeolite frameworks. (b) The effect of Gaussian blurring on the atomic structure of CHA. APT data has more blurring than these cases for zeolites. (c) A C atom map with a 10 at.\% iso-concentration surface for the coked zeolite ZSM-5. The black spheres represent clustered C atoms. (d) A proximity histogram of $\mathrm{Al}$ rich regions in aged zeolite $\mathrm{Cu}-\mathrm{ZSM}-5$ showing a stoichiometry close to $\mathrm{Al}_{2} \mathrm{CuO}_{4}$. The $\mathrm{Al} / \mathrm{Cu}$ ratio stabilizes at $\sim$. (b) and (c) are modified from references [2] and [3], respectively.

\section{References}

[1] J.E. Schmidt et al., J. Am. Chem. Soc., (2018), 140, p. 9154.

[2] J.E. Schmidt et al., Angew. Chem. Int. Ed., (2016), 128, p. 11339.

[3] J.E. Schmidt et al., Angew. Chem. Int. Ed., (2018), 57, p. 10422.

[4] J.E. Schmidt et al., Nat. Commun., (2017), 8, no. 1666.

[5] Research was conducted at ORNL's Center for Nanophase Materials Sciences (CNMS), which is a U.S. DOE Office of Science User Facility. 